Documentation et bibliothèques

DOCUMENTATION BIBLIOTHEQUES

\title{
L'école et le plaisir de lire
}

\section{The School and the Pleasure of Reading}

La escuela y el placer de leer

\section{Monique Lebrun}

Volume 39, numéro 3, juillet-septembre 1993

URI : https://id.erudit.org/iderudit/1028746ar

DOI : https://doi.org/10.7202/1028746ar

Aller au sommaire du numéro

\section{Éditeur(s)}

Association pour l'avancement des sciences et des techniques de la documentation (ASTED)

ISSN

0315-2340 (imprimé)

2291-8949 (numérique)

Découvrir la revue

Citer cet article

Lebrun, M. (1993). L'école et le plaisir de lire. Documentation et bibliothèques, 39(3), 111-116. https://doi.org/10.7202/1028746ar

\section{Résumé de l'article}

L'école a mauvaise presse dès qu'il s'agit de la lecture. On l'accuse de préférer les recettes et les cheminements stéréotypés à une véritable ouverture sur l'univers imaginaire. Nous tenterons de démontrer, en adoptant des points de vue empruntés à la didactique, à la théorie littéraire et à la psychologie que s’il en a longtemps été ainsi, on peut parler, au moins depuis dix ans, d'une ouverture de plus en plus grande sur les besoins des lecteurs, et partant, sur leurs attitudes. Il faut toutefois compter sur des réformes de programmes, et surtout sur un renouvellement des pratiques pédagogiques.
Tous droits réservés (c) Association pour l'avancement des sciences et des techniques de la documentation (ASTED), 1993
Ce document est protégé par la loi sur le droit d'auteur. L'utilisation des services d'Érudit (y compris la reproduction) est assujettie à sa politique d'utilisation que vous pouvez consulter en ligne.

https://apropos.erudit.org/fr/usagers/politique-dutilisation/ 


\title{
L'école et le plaisir de lire
}

\author{
Monique Lebrun \\ Département des sciences de l'éducation \\ Université du Québec à Montréal
}

L'école a mauvaise presse dès qu'il s'agit de la lecture. On l'accuse de préférer les recettes et les cheminements stéréotypés à une véritable ouverture sur l'univers imaginaire. Nous tenterons de démontrer, en adoptant des points de vue empruntés à la didactique, à la théorie littéraire et à la psychologie que s'il en a longtemps été ainsi, on peut parler, au moins depuis dix ans, d'une ouverture de plus en plus grande surles besoins des lecteurs, et partant, surleurs attitudes. II faut toutefois compter sur des réformes de programmes, et surtout sur un renouvellement des pratiques pédagogiques.

\section{The School and the Pleasure of Reading}

When it comes to reading, the school has a poor image. Some claim that the school prefers quick fixes and pre-established pathways ratherthan atrue awakening of the imagination. Drawing from the fields of didactics, literary theory and psychology, we will establish that if this situation has persisted for approximately ten years, a new awareness of the needs and attitudes of readers is now emerging. Pedagogical reform and new teaching methods must also be put to the task.

\section{La escuela y el placer de leer}

Cuando se trata de la lectura se hace mala propaganda a la escuela. Se le acusa preferir las maneras de enseñanza estereotipadas a una verdadera perspectiva sobre el universo imaginario. Trataremos de demostrar, adoptando los puntos de vista tomados de la didáctica, la teoría literaria y la psicología que, si asi lo fue desde mucho tiempo, podemos hablar, a lo menos desde hacen diez años, de una apertura más y más grande sobre las necesidades de los lectores, y a partir de sus actitudes. Sin embargo, debemos contar con las reformas de programas, y sobre todo de una renovación de las prácticas padagógicas.
II me semble que quand le système scolaire joue le rôle qu'il joue dans nos sociétés, c'est-à-dire quand il devient la voie principale ou exclusive de l'accès à la lecture, et que la lecture devient accessible pratiquement à tout le monde, je pense qu'il produit un effet inattendu. Ce qui m'a frappé dans les témoignages d'autodidactes qui nous ont été rapportés, c'est qu'ils témoignaient d'une sorte de besoin de lecture, que d'une certaine façon, l'école détruit pour en former un autre. Ily a un effet d'éradication du besoin de lecture comme besoin d'information: celui qui prend le livre comme dépositaire de secrets (...) qui tient le livre comme un guide de vie (...). Je pense que le système scolaire a cet effet paradoxal de déraciner cette attente (...) de prophétie au sens wébérien de réponse systématique à tous les problèmes de l'existence ${ }^{1}$
Depuis deux décennies, pour le moins, les Cassandre de l'opinion publique dénoncent une désaffection pour la lecture qui, dit-on, proviendrait des lacunes de l'école. Les programmes feraient fi du plaisir de lire, cantonneraient écoliers et élèves dans la lecture de textes courts et peu nombreux. II n'est jusqu'aux enseignants que l'on accuse d'utiliser des stratégies sans imagination pour susciter ou évaluer la compréhension en lecture. Qu'en est-il, exactement?

Nous nous proposons de démontrer que les enjeux de la lecture à l'école sont variés et moins imperméables au plaisir de lire qu'il n'y paraît. Notre perspective sera à la fois historique, littéraire et psychopédagogique. En effet, on ne peut bien parler de la lecture sans évoquer l'évolution récente du contexte scolaire, ni sans préciser les mutations du concept même de compréhension en lecture, tributaire à la fois des contributions de la psychologie cognitive et des théories littéraires. Inspirée avant tout par le contexte québécois, notre réflexion tient également compte des avancées de la recherche européenne et américaine. Après avoir évoqué la conception de la lecture ayant cours à l'école antérieurement aux années soixante, nous nous pencherons sur les bouleversements entraînés par une pédagogie techniciste, pour enfin aborder les voies prometteuses de la réforme.

\section{La primauté des pédagogues}

Nous laisserons ici de côté, volontairement, les premiers apprentissages, de type «décodage», pour nous intéresser aux jeunes lecteurs qui, à partir de l'âge de huit ans environ, maîtrisent suffisamment les rudiments pour pratiquer la

1. Pratiques de lecture sous la direction de Roger Chartier, Paris, Rivages, 1985, p. 227-228. 
lecture de façon relativement autonome. Jusqu'à l'entrée en trombe de la linguistique, de la sémiotique et de la sociologie des phénomènes culturels dans le domaine de la compréhension en lecture soit jusqu'aux années soixante, le pédagogue choisissait seul à la fois les textes et les différentes stratégies d'exploration du sens. Voyons certaines tendances.

Ainsi que nous le mentionnent Chartier et Hébrard ${ }^{2}$, c'est une fois les rudiments du déchiffrage acquis que l'élève peut entrer de plain-pied dans la «lecture expliquée». Ce concept, codifié grâce aux travaux de Gustave Lanson ${ }^{3}$, apparaît au début du siècle: on y mêle explications sur la langue et sur les idées d'un texte en un exercice oral et collectif. Derrière le questionnement se profile l'idée d'un sens immanent au texte, connu de l'enseignant et qu'une maïeutique appropriée doit permettre à l'élève de découvrir. Le cheminement vers le sens par le recours au questionnement deviendra, surtout dans les petites classes, convenu, sans surprise, voire stéréotypé. Cela est d'autant plus étonnant que la «lecture expliquée» surgit au moment où, dans les années vingt, la pédagogie active prend son essor, sous l'influence des Claparède, Decroly et Dewey.

Le peu d'études dont nous disposons sur la place historique de la lecture dans le cadre scolaire québécois de cette époque nous permet néanmoins de conclure en la prégnance du modèle lansonien. Dans le premier ouvrage québécois de méthodologie destiné aux enseignants, Ross ${ }^{4}$ soulève les problèmes inhérents à la lecture expliquée: celle-ci ne doit pas se substituer à la lecture courante, mais s'orienter vers une analyse littéraire mise à la portée des élèves, surtout aux cycles supérieurs. Par elle, l'enseignant vise la formation intellectuelle et morale des élèves. Si Ross parle de l'enseignement public, on peut penser que des principes identiques se retrouvent, à la même époque, dans les classes de lettres du cours classique. Dans ses travaux sur la question, l'équipe du (CRELIQ) ${ }^{5}$ abonde en ce sens. Ses collaborateurs examinent le discours institutionnel tenu durant cent cinquante ans sur l'exploitation du texte littéraire à l'école à travers les programmes, les anthologies et même les travaux d'élèves. Le texte littéraire, y affirme-t-elle, n'était pas étudié pour lui-même, mais pour sa valeur de formation en lecture et écriture, en logique et en morale. Les deux exercices privilégiés étaient l'analyse de texte et la dissertation. On regroupait habituellement les textes choisis dans des anthologies révélatrices de l'axiologie sur le discours littéraire: "l'humanisme recherché (était) fait de connaissances étendues, de jugements avisés, de bon goût et de rectitude morale $(\ldots)^{6} \%$. Bien lire conduisait à bien penser et à bien écrire. Le discours idéologique était clos, la lecture était monosémique, dans la droite ligne des attentes institutionnelles. Qui ne se souvient de l' «enfer» des bibliothèques de collège, lieu où l'on enfermait à double tour les livres à l'index réputés offenser la pudeur des jeunes âmes, et dont on peut retrouver un célèbre avatar dans $L e$ libraire de Bessette! II faudrait ici rappeler dans quelle crainte les éducateurs, influencés par l'attitude de l'Église, ont tenu le genre romanesque, et ceci, presque jusqu'à l'entre-deux guerres. Celui-ci leur semblait la voie ouverte à l'immoralisme et à la rêverie frivole.

On l'a vu, il est abondamment question de texte littéraire dans l'exercice de lecture expliquée, mais il s'agit d'un texte sous forme de morceaux choisis ad usum delphini. Il est important que le texte porte la signature d'un auteur connu: en retour, l'école officialise son appartenance à un courant littéraire et le consacre comme mentor des générations en formation. Pour reprendre la célèbre boutade de Barthes: "La littérature, c'est ce qui s'enseigne!». L'approche du texte est, pour emprunter une expression moderne, "externe», c'està-dire incluant un large recours à l'histoire littéraire, à sa périodicité, à la nomenclature des grands auteurs, de leurs thèmes et filiations. Il est souvent difficile, souligne Chervel $^{7}$ de définir une méthode d'approche purement littéraire qui se distingue de celles des sciences humaines, puisque l'explication de texte mêle allègrement histoire, philosophie et religion. D'ailleurs, le professeur de lettres, au secondaire, enseigne souvent ces autres matières.

Avant 1960 donc, les pratiques scolaires, pour soucieuses qu'elles aient été de la formation intellectuelle et morale des élèves par les textes, n'encourageaient pas la lecture extensive des textes, l'appropriation personnelle, en somme, le plaisir de lire. Celui-ci se découvrait au hasard des lectures de vacances, dans des ouvrages de «distributions des prix» et autres oeuvres approuvés: on pense ici au Guide général des lectures de l'abbé Bethléem en France, et à l'imprimatur donné par nos évêques, jusqu'à une date récente, à des ouvrages de fiction pour la jeunesse tenant souvent de l'hagiographie. Heureusement, les années soixante allaient ébranler ce bel édifice!

\section{L'ère des scientifiques du texte}

L'école est une institution beaucoup plus ouverte qu'on ne le croit aux modes d'une société, du moins depuis quelques décennies. Durant les années soixante, en raison des avancées conjointes de la linguistique de texte (grammaire de texte) et de la psycho-pédagogie de la communication, on assiste à un revampage des programmes scolaires dans le domaine de la lecture. Déjà, durant les années cinquante, les premières enquêtes sur la lecture avaient soulevé le problème du peu d'utilitarisme social des pratiques de lecture et mis face à face les tenants de la culture classique et ceux du pragmatisme. Désormais, le couple lecture expliquéedissertation tendra à céder la place au trio fiche de lecture-exposé-discussion. Les années soixante voient également la mise en place d'une problématique de l'échec en lecture que d'aucuns ${ }^{8}$ s'empresseront $^{\prime}$ de lier à l'origine sociale.

2. A.M. Chartier et J. Hébrard, Discours sur la lecture (1880-1980), Paris, Centre GeorgesPompidou, 1989.

3. Gustave Lanson, "Quelques mots sur l'explication de texte», Études françaises, janvier 1925, 38-57.

4. François-Xavier Ross, Pédagogie théorique et pratique, $3^{\mathbf{e}}$ ed., Québec, Imprimerie Charrier et Dugal, 1924.

5. J. Melançon et al., Le discours d'une didactique. La fonction du littéraire dans l'enseignement classique au Québec (1852-1867), Québec, Nuit Blanche, 1988. (Collection Recherche, no. 1)

6. Ibid., 17

7. A. Chervel, Les auteurs français, latins et grecs au programme de l'enseignement secondaire de 1800 à nos jours, Paris, Institut national de recherches pédagogiques et Publications de la Sorbonne, 1986.

8. Pierre Bourdieu et Jean-Claude Passeron, Les héritiers, Paris, Éditions ouvrières, 1992. 
Les changements de la conception scolaire de la lecture que nous illustrerons par le biais de certains programmes manifestent les soubresauts culturels d'une société qui rêve de pédagogie active tout en codifiant de plus en plus l'entrée dans le texte, qui se laisse séduire par le technicisme en éprouvant la nostalgie de l'humanisme classique. Les travaux des sémiologues répandent l'idée de la lecture perçue comme un pur exercice d'intelligence (cf les schémas actantiels, carrés sémiotiques et autres exercices de haute voltige). Parallèlement, l'avant-garde psycho-pédagogique enseigne qu'il faut tenir compte du savoir pré-construit du lecteur, privilégier les situations «vraies», la pédagogie de la découverte. Les linguistes pénètrent à l'école par le biais de la linguistiqueappliquée et répandent l'idée des habiletés langagières décontextualisées, mais facilement mesurables quant à leur atteinte (par exemple, le vocabulaire, ou encore, les tournures syntaxique). Bref, l'enseignant de la base se sent envahi: tous lui proposent des «stratégies» de lecture. II lui faut à la fois former des lecteurs polyvalents et exalter, autant que faire se peut, le rapport ludique au livre.

En France et en Belgique, les années soixante et soixante-dix sont marquées par l'interrogation sur la place de la lecture «lettrée» à l'école, surtout dans un contexte où la culture médiatique se fait omniprésente. Le clivage devient de plus en plus flagrant entre le primaire, où le texte est réduit à sa fonction de modèle éventuel pour la rédaction, et le secondaire qui ouvre sur le littéraire. Comme le mentionne Marcoin ${ }^{9}$, pareille position entraîne, pour le primaire, la tentation de la quotidienneté, la réduction aux apprentissages instrumentaux.

Dans son enquête auprès des élèves français de la classe de sixième (l'équivalent des débuts du secondaire au Québec), Béguin ${ }^{10}$ découvre que pour eux la lecture est surtout une activité de contrôle et de sélection: on se résigne à lire pour soitdisant progresser en compréhension. $Y$ apparaît l'importance de l'enseignement qui motive par ses choix et ses attitudes. Comme le dit Gratiot-Alphandry:

Àl'âge de la préadolescence, la lecture connaît son épreuve de vérité. Les programmes scolaires imposent de lire pour apprendre et aussi hélas pour expliquer, c'est-à-dire analyser, dépiauter, décortiquer; le livre, l'auteur deviennent squelette, planche anatomique, et la circulation du sang, le souffle de la vie ont disparu. $\dot{A}$ ce moment,toute une image de la personne dans sa relation au livre est en cause et menacée. Tout ce que le livre apporte à ce niveau: l'évasion, l'identification, l'information, risquent de disparaître sous ces analyses exigeantes. Un auteur, même contemporain, peut-il encore susciter les mêmes enthousiasmes ou les mêmes désaveux quand il est "mis au programme»? Et n'y a-t-il pas là quelque façon d'attenter à cette relation exceptionnelle entre le lecteur et l'auteur oùil est besoin d'une certaine discrétion ${ }^{11}$.

On le sait, ce qui attire d'abord l'élève, c'est l'histoire qui lui permet de vivre des aventures par procuration. Pour éprouver du plaisir, il faut pouvoir lire longuement, sans contrainte de règles, de umises en situation», d'évaluations. Au Québec, le coup de barre est donné par le Rapport Parent ${ }^{12}$, en 1964, et par ce qu'on a appelé le programme cadre de français. Le Rapport Parent parle abondamment de la réforme des pratiques de lecture du texte littéraire et leur assigne quatre buts: développer l'intelligence et la sensibilité, favoriser la connaissance de la langue, contribuer à l'enrichissement moral, assurer un contact humanisant avec les meilleures oeuvres qu'ait produit l'esprit humain. On le voit, l'objectif éthique de jadis est désormais assorti d'objectifs esthétiques et culturels. On prône la mémorisation de texte, la lecture obligatoire d'un minimum de trente livres par année (sic!) choisis dans une liste.

Quelque cinq ans plus tard apparaît le programme cadre de français ${ }^{13}$ qui remplace les programmes "catalogues» des décennies précédentes. Au chapitre de l'apprentissage en lecture, ces programmes n'imposent pas de méthode particulière; tout au plus recommandent-ils le recours à la lecture expliquée (ex.: la recherche des idées principales et secondaires) accompagnée d'un travail de rédaction auquel on greffe des exercices de vocabulaire et d'orthographe. C'est contre eux qu'a tonné le Frère Untel dans ses célèbres Insolences ${ }^{14}$
Les 70 pages du programme de français au primaire de 1959 étaient incluses dans un fort volume de 695 pages; avec le programme-cadre de 1969 , on passe à une mince plaquette de seize pages. Pour la première fois, mais non la dernière dans les programmes, le terme «communication» prend la vedette: l'enseignement doit apprendre à ses élèves à maîtriser leur langue afin de mieux communiquer avec autrui. Tant au primaire qu'au secondaire, on doit désormais développer les quatre savoirs: savoir parler, savoir écouter, savoir lire et savoir écrire. On veut favoriser la réflexion et la création linguistiques. On insiste sur l'influence du milieu dans l'apprentissage de la langue maternelle et sur le fait qu'il faut tenir compte de la spontanéité de l'expression de l'enfant. On affirme que «le travail scolaire porte sur le français parlé d'abord, sur le français écrit ensuite» ${ }^{15}$ : : il faut sans doute voir là l'influence d'une certaine psychologie de la non-directivité propre à l'époque et des méthodes d'enseignement de la langue seconde. La connaissance du code est subordonnée à l'expression personnelle: la leçon de français n'est plus centrée sur le contenu, mais sur l'élève. La leçon de lecture est réduite à sa portion congrue d'autant plus qu'on décrète presque simultanément la mort du manuel, son remplacement par les polycopiés et qu'on introduit pour la première fois les textes dits «utilitaires» (ex. : la lettre, le curriculum vitae, l'interview).

9. F. Marcoin, À l'école de la littérature, Paris, Éditions ouvrières, 1992.

10. Albert Béguin, Lire-écrire; pratique nouvelle de la lecture au collège, Paris, L'École, 1982.

11. H. Gratiot-Alphandry, «Le rôle de la lecture dans la formation de l'enfant et de l'adolescent» in $L e$ pouvoir de lire, sous la direction de J. Jolibert et R. Gloton, Paris, Casterman, 1975, p. 38.

12. Commission royale d'enquête sur l'enseignement dans la province de Québec, Rapport, Québec, 1966. Volume 3: Les programmes d'études.

13. Québec, Ministère de l'Éducation, Direction générale de l'enseignement élémentaire et secondaire, Programme cadre de français. Langues et littérature, Québec, 1969.

14. Jean-Paul Desbiens, Les insolences du Frère Untel, Montréal, Éditions de l'homme, 1959.

15. Québec, Ministère de l'Éducation, Direction générale de l'enseignement élémentaire et secondaire. Programme cadre du français ...., p. 4 
La lecture occupe une place plus importante dans les programmes de français de $1979^{16}$. Comme dans le programme de 1969, le maître-mot est «communication», mais on y joint des concepts complémentaires: les discours définis à la façon de Jakobson et la technique de l'objectivation, qui rejoint les antiques techniques de révision, de relecture et de réécriture. La terminologie change: on n'écrit plus, on ne parle plus, on fait une "production orale ou écrite»; on ne s'interroge plus sur l'auteur d'un texte, mais sur l'émetteur d'un discours; on ne cherche plus un message, mais bien une intention de communication. De transmetteur de connaissances qu'il était, l'enseignant devient un animateur.

Au primaire, on recommande l'étude des discours expressifs, informatifs, incitatifs, et ludiques, discours que l'on retrouve également au secondaire. On les analyse dans une perspective textuelle qui recourt, sans les nommer, aux théories sémiotiques. Le discours de type littéraire occupe une place timide, mais l'histoire littéraire est totalement oblitérée, bien qu'on mentionne, dans les objectifs généraux du programme, la nécessité de situer les discours dans une perspective socio-culturelle. Les textes sont généralement courts et la démarche, traditionnelle, même dans l'exploitation du schéma de la communication. Cette pédagogie reste marquée par la priorité donnée au contenu informationnel du texte. II s'agit là d'une tendance née de la place considérable prise par la psychologie cognitive dans l'enseignement des langues. Les pratiques inspirées de cette école visent à ce que le lecteur ne retire de la page de texte que ce que l'auteur y a déposé. Les opérations mentales et les attitudes que l'on demande au lecteur de développer sont invariables, que le texte soit narratif ou informatif. On amenuise de la sorte les capacités interprétatives de l'élève et, en voulant faire de la lecture une activité "efficace», on rend celle-ci mécaniste. On va même jusqu'à calibrer le vocabulaire et les structures de phrase selon les âges.

\section{L'ouverture au plaisir du texte: mission impossible?}

Les écrivains et spécialistes du texte ont été les premiers à s'inquiéter de la dérive utilitariste et mécaniste de la leçon de lecture, plus particulièrement de la lecture littéraire, comme en témoigne un colloque sur l'enseignement de la littérature tenu à Cerisy en 1970 réunissant, en autres, Doubrovsky, Greimas et Barthes. On y a reconnu la primauté du rôle de l'enseignant, médiateur de culture et transmetteur du contexte socio-culturel du texte. On y a évoqué les pratiques soucieuses de polysémie et de pluralisme axiologique. On y a surtout situé l'expérience littéraire dans le cadre plus vaste de l'expérience esthétique, qui est de l'ordre non de l'intellect, mais de l'affect. Pour cela, il faut, non pas tant parler de l'oeuvre, mais laisser parler l'oeuvre:

Cette voix (celle de l'écrivain), le professeur, s'il est digne de sa fonction, lui prête la sienne. Illui offre sa personne. Dès lors, support de désir et de répulsion, lieu et chair de l'affect, il se montre, s'exhibe. Toute estrade est un tréteau. Acteur, professeur: histrions par qui sincarne le verbe ${ }^{17}$.

Quelque vingt ans plus tard, on retrouvera le même point de vue, la même ferveur chez Pennac ${ }^{18}$, pour lequel l'enseignant est une «marieuse» entre le texte et l'élève, et qui prône, pour ce faire, la lecture expressive à voix haute à la classe d'oeuvres entières et bien choisies. Le ton n'est pas toujours aussi poli, cependant, certains «littéraires» n'hésitant pas à devenir des polémistes d'occasion, dénonçant l'influence néfaste des manuels de lecture:

surdidactisés par suite d'une visualisation excessive au moyen de l'image supportant le texte et de l'encadrement multicolore de passages textuels importants. Devant les textes aménagés de cette manière, le lecteur - enfant scolaire ou étudiant - finit par ne plus avoir besoin pratiquement de lire méticuleusement, car il n'y a presque plus rien à lire, à découvrir individuellement dans le texte ${ }^{19}$.

Les littéraires en ont donc contre la vision imposée, l'absence de processus de découverte, les «impératifs d'ordre mimétique ou spéculaire» ${ }^{20}$ d'une pédagogie où tout est prévu. Ils répètent qu'il n'y a pas de décodage canonique des oeuvres, de "fausse» lecture, même si, bien sûr, les lectures ne se valent pas toutes.
Plus que jamais, en cette fin de siècle, apparaît la dichotomie entre la lecture fonctionnelle et la lecture esthétique, la seule qui soit véritablement formatrice. II semble bien que, par consensus social, on veuille reléguer l'école à la première, comptant sur la famille et sur les bibliothèques pour mousser la seconde. Suite à la réflexion des spécialistes du texte, certains pédagogues se sont pris au jeu et ont commencé à revendiquer, pour l'école, la mission d'inciter au plaisir de lire. Ils ont été les premiers à revendiquer l'élargissement du corpus, l'application des méthodes reliées à l'intertextualité, la prise en compte de méthodes interactives où l'élève soit un agent de sa formation par la lecture, la lecture d'oeuvres complètes et exigeantes, la sollicitation du monde imaginaire de l'élève, l'intégration des pratiques de lecture à un projet. Nous expliquerons maintenant leur point de vue qui comporte, on le devinera, une redéfinition de ce qu'est le processus de compréhension en lecture.

Les nouveaux «pédagogues de la lecture», ainsi que nous pourrions les nommer, s'entendent sur des postulats communs. Pour eux, la véritable expérience de lecture passe par le texte littéraire (ou narratif). Celui-ci possède une dimension existentielle irréductible au savoir ou savoir faire développés par une école technicisante et rationnalisante. Les stratégies de lecture à développer doivent toucher tout autant le coeur que la raison, sous peine de provoquer la désaffectation

16. Québec, Ministère de l'Éducation, Programme d'études. Primaire. Français, Québec, 1979; Programme d'études. Français, langue maternelle (secondaire), Québec, 1980.

17. L'enseignement de la littérature. Langages nouveaux, pratiques nouvelles pour la classe de français, sous la direction de S. Doubrovsky et T. Todorov, Bruxelles, De Boeck-Duculot, 1981.

18. Daniel Pennac, Comme un roman, Paris, Gallimard, 1992.

19. R. Reisinger, "Le point de vue du professeur", in La lecture littéraire, sous la direction de $\mathrm{M}$. Picard, Paris, Clancier-Guénaud, 1987, p. 278.

20. M. A. Seixo, «L'activité de la lecture: travail et pédagogie", in La lecture littéraire, sous la direction de M. Picard, Paris, Clancier-Guénaud, 1987, p. 299. 
du livre. Enfin, ces pédagogues admettent que l'on ne peut tout évaluer, même en lecture scolaire. Nous évoquerons ci-après deux grands courants: le courant socioanthropologique européen et le courant de la réception esthétique américain.

Le courant socio-anthropologique européen s'est laissé inspirer, dans son approche du texte littéraire par les travaux des philosophes Jankélévitch, De Certeau etRicoeur, qui prônent une logique ouverte aux risques et une épistémologie de l'implication plus que de l'application. Ainsi, pour De Certeau ${ }^{21}$, l'école en est venue à privilégier la forme au détriment du contenu, d'où un enseignement normalisé et un point de vue sur le texte comme objet de consommation à rendre digestible en «organisant» des attentes, en "ordonnant» des codes de perception.

Selon De Certeau, la vraie lecture ne peut être que plurielle, basée sur la créativité transgressive du lecteur. On retrouve ces positions, entre autres, dans les travaux de l'école de didactique du français de Louvain ${ }^{22}$ et dans l'approche socio-culturelle des animateurs de l'équipe de Pratiques ${ }^{23}$. Essentiellement, les pédagogues de cette tendance visent l'appropriation personnelle du texte et le va-et-vient entre l'affect et l'intellect. L'équipe de Louvain est particulièrement sensible à la dichotomie entre le «playing» et le "game» de Winnicott ${ }^{24}$, distinction reprise et développée dans le cadre des études littéraires par Picard ${ }^{25}$. Sommairement dit, le «playing» est le jeu ouvert, qui introduit une lecture participative et émotionnelle. La référentiation est externe: c'est la mimésis ou fonction référentielle. Quant au «game», c'est la lecture «construite», objectivante, réglée. La référentiation est alors interne et intertextuelle: c'est le sémiosis.

Quant à l'approche de l'équipe de Pratiques, elle doit beaucoup aux avancées des sociologues du littéraire, surtout celle de Leenhardt ${ }^{26,27}$. Celui-ci a proposé de définir trois modalités de lecture: la modalité phénoménale, ou descriptive, qui est distanciation du texte, la modalité émotionnelle ou identificatoire et enfin, la modalité intellective, la plus valable, qui opère la jonction des deux précédentes. Leenhardt insiste sur le fait que l'école est une instance de légitimation de la lecture.
Les travaux du groupe Pratiques ${ }^{28}$ soutiennent, dans la même foulée, que le modèle légitimé par l'école ne correspond pas toujours aux références culturelles des élèves d'aujourd'hui et que l'école doit permettre l'apprentissage des médiations culturelles en s'intéressant, entre autres, au circuit auteur-livre-éditeur et à la paralittérature.

Concrètement, dans la salle de classe, les positions ci-haut mises de l'avant débouchent sur une redéfinition de la compréhension de texte qui laisse la place au lecteur et à son interprétation, sur le syncrétisme du corpus, en vertu duquel même les textes paralittéraires sont accueillis, sur la mise en évidence de la dimension intertextuelle, sur la notion de «parcours» de lecture, où chaque activité constitue une progression par rapport à la précédente. On vise à faire lire des textes "premiers», fondateurs d'une culture, à enrichir les référents culturels de l'élève. La lecture n'est plus «expliquée», mais «méthodique»: il s'agit d'enrichir le «dictionnaire de base» (lexique et grammaire), d'aider à la reconnaissance des clichés et stéréotypes, de développer les référents mythico-religieux, d'enrichir le code symbolique, d'acheminer vers la reconnaissance des marques idéologiques. Des revues spécialisées ${ }^{29}$ donnent d'ailleurs à l'enseignant des suggestions en ce sens.

Outre ce courant européen de réforme, nous avons plus haut fait état d'un courant américain de la réception esthétique. II s'agit de l'adaptation faite par les didacticiens des théoriciens de l'esthétique de la réception ${ }^{30}$ dont les plus connus, à partir des années soixante, ont été Jauss, Iser et Eco. Nous ne pouvons nous attarder, dans le cadre de cet article, à l'exposé de leurs positions. Qu'il suffise de dire que , pour eux, le lecteur est, à l'égal de l'auteur, créateur de texte à cause de ses capacités interprétatives. Conjuguées avec les travaux de la psychologie cognitiviste, particulièrement le constructivisme appliqué à la compréhension en lecture, les thèses des tenants de l'esthétique de la réception ont fait du lecteur un roi. La pédagogueaméricaine Rosenblatt ${ }^{31} a$, quant à elle, contribué à vulgariser l'approche de l'esthétique de la réception en classe par ce qu'elle appelle l'approche transactionnelle, selon laquelle le lecteur interagit avec le texte. On trouvera particulièrement
21. M. de Certeau, $L$ 'invention du quotidien, $1-L$ 'art de faire. Paris, Gallimard, 1990. (Folio Essais)

22. Il s'agit de l'Unité de didactique du français de l'Université catholique de Louvain animée par Pierre Terlès et dont les publications (chez Didier /HMH) sont nombreuses, particulièrement sur l'expertise des genres courts en classe de français. Voir J.-L. Dufays, Stéréotypes et lecture. Propositions pour une théorie et une didactique de la réception littéraire, Louvain, Faculté de philosophie et lettres, 1991. Thèse de doctorat. Pierre Yerles et M. Lits, «Pour une didactique de la littérature», Dialogues et cultures, no. 36, 1992 , 107-118.

23. «Pratiques» est un collectif de militants d'un enseignement rénové du français publiant, à Metz, une revue et des documents didactiques.

24. D. Winnicott, Jeu et réalité. L'espace potentiel, Paris, Gallimard, 1971.

25. M. Picard, La lecture comme jeu, Paris, Éditions de minuit. 1986.

26. J. Leenhardt, «Les instances de la compétence dans l'activité lecture», in La lecture littéraire, sous la direction de M. Picard, Paris, ClancierGuénaud, 1987, p. 302-311.

27. «Les effets esthétiques de l'oeuvre littéraire: un problème social»), in Pour une sociologie de la lecture. Lectures et lecteurs dans la France contemporaine, sous la direction de Martine Poulain, Paris, Éditions du Cercle de la Librairie, 1988, p. 59-79.

28. A. Petitjean, «Pour une didactique de la littérature», in Perspectives didactiques en français, sous la direction de J.-F.Halté, Metz, Centre d'analyse syntaxique de l'Université de Metz, 1990.

29. En plus de la revue Pratiques, déjà citée, on peut mentionner La nouvelle revue pédagogique.

30. W. Iser, L'acte de lecture: théorie de l'effet esthétique, Bruxelles, Mardaga, 1978. H. R. Jauss, Pour une esthétique de la réception, Paris, Gallimard, 1978. Umberto Eco, Lector in fabula, Paris, Grasset, 1985.

31. L M. Roseblatt, "The Aesthetic Transaction», Journal of Aesthetic Education, vol. 20, no. 4 (1986), 122-128.

Litterature as Exploration, Londres, Heinemann, 1983.

"The Literary Transaction: Evocation and Response», Theory into Practice, vol. 21 , no. 4 (1982), 268-277.

The Reader, The Text, The Poem. The Transactional theory of the literary World, Carbondale, Ill., Southern Illinois University Press, 1978 
féconde son opposition entre lecture "efférente» et lecture «esthétique». Alors que la première se contente d'extraire des informations du texte ou de les inférer par un téléguidage s'apparentant à un contrat figé de lecture, la seconde vise la reconstruction du sens pour soi, en une lecture-événement où la subjectivité s'unit aux capacités raisonnantes. On retrouve les idées de Rosenblatt dans les travaux actuels de Grave et Atwell ${ }^{32}$ sur le «journal dialogue», formule où l'enseignant personnalise, par un échange écrit soutenu et individuel avec chaque élève, sa perception des relations entre l'élève et le texte.

Au Québec, le groupe LALA (Lecture accompagnée, littérature apprivoisée) ${ }^{33}$ travaille depuis 1990 selon une approche conjuguant les théories de Rosenblatt et celles de Langer ${ }^{34}$, sur les niveaux d'imprégnation par le texte (c'est-à-dire: contact, immersion, projection personnelle et distanciation). Cette équipe mène, depuis 1990, une véritable recherche-action, de concert avec les enseignants et conseillers pédagogiques de cinq commissions scolaires. Elle a construit un «modèle binaire d'appropriation du texte en lecture» basé sur la théorie de la réception, de même qu'une grille d' «indicateurs d'apprentissage» en lecture qui vient préciser les niveaux de Langer. Elle a surtout précisé une démarche pédagogique toute entière centrée sur le plaisir de lire grâce à des formules pédagogiques de type interactif où l'enseignant met en veilleuse son rôle officiel et suscite des réactions personnalisées, de même qu'à un corpus ambitieux susceptible de provoquer de véritables expériences esthétiques.

\section{Conclusion}

On le sait, toute une frange de l'information, de la culture et du plaisir passe aujourd'hui par d'autres médias que le livre: branchés qu'ils sont sur les écrans cathodiques, les enfants de cette fin de siècle deviennent imperméables aux mots. D'autre part, dans une époque d'instantanéité, les livres entiers les effraient; plus encore, les questionnements stéréotypés sur des textes calibrés et manipulés les ennuient. Ce que l'école doit viser, pour permettre l'atteinte par tous du plaisir de lire, c'est le changement de ses corpus et de ses méthodes. Pour développer l'appétence textuelle, l'enseignant doit insérer le livre dans un réseau socio-culturel, oublier le souci de la microévaluation, se poser lui-même comme en état de recherche. De plus en plus de pédagogues le comprennent et tendent à infléchir en ce sens les situations de lecture qu'ils créent. La réforme actuelle des programmes québécois de français du primaire et du secondaire, où le texte littéraire et les objectifs liés au plaisir de lecture prennent de plus en plus de place devraient nous porter à croire qu'un nouvel humanisme commence à poindre, après une lente gestation.

32. D. Graves, Experiment with Fiction, Porthsmouth, N.H., Heinemann, 1983. N. Atwell, In the Middle: Writing, Reading and Learning with Adolescents. Porthsmouth, N.H., Heinemann, 1987.

33. Monique Lebrun et $M$. Le Pailleur, «De la lecture efférente à la lecture esthétique des récits» in $L a$ lecture et l'écriture: enseignement et apprentissage, sous la direction deC. Préfontaine et Monique Lebrun, Montréal, Logiques, 1992, p. 183-200.

34. Monique Lebrun, «Des formules pédagogiques nouvelles pour des textes narratifs au primaire", in $L$ 'hétérogénéité des apprenants, un défi pour la classe de français, sous la direction de Monique Lebrun et M.-C. Paret, Neuchatel, Delachaux et Niestlé, 1993, p. 188-193.

\section{Prin spécial de Lancement!}

\section{UORDS \& PHRASES}

Words \& Phrases est l'ouvrage canadien que vous utiliserez quotidiennement afin de connaître l'interprétation judiciaire donnée aux termes et locutions provenant de TOUS les domaines de droit, et ce rapidement et facilement! L'ouvrage contient à la fois des expressions d'origine statutaire et de common law, issues de décisions canadiennes rapportées jusqu'à la fin de l'année 1992.

Conçu pour vous aider à obtenir gain de cause lors d'un litige ou à rédiger des documents légaux sûrs, l'ouvrage comporte

- 50,000 entrées;

- une liste alphabétique de termes et locutions considérés par les cours et tribunaux canadiens - utilisez-la comme un dictionnaire;

- le nom du juge cité et sa juridiction;

\section{interprétés par les cours et tribunaux canadiens}

- des références incluant un renvoi précis à la page d'où provient l'entrée;

- un système de renvois élaboré vous permettant de repérer TOUTES les entrées pertinentes;

- 8 volumes reliés à couverture rigide.

De plus, Words \& Phrases est mis à jour annuellement grâce à un supplément cumulatif à couverture souple. Vous serez donc constamment à jour en ce qui concerne les termes et locutions interprétés judiciairement au Canada.
0-459-54143-9 Septembre 19938 volumes à couverture rigide $950,00 \$$ (prix du catalogue)
Prix spécial de lancement - 875,00\$
Frais de port et de manutention en sus.
Prix sujet à changement sans préavis, ainsi qu'aux taxes applicables.

POUR COMMANDER - APPELEZ SANS FRAIS AU

$1-800-387-5164$

À TORONTO: (416) 609-3800 Demeondex lo teléphoniste n. 846

PAR TÉLÉCOPIEUR: (416) 298-5094 (24 heures)

OU ÉCRIVEZ Ȧ: CARSWELL,1, Corporate Plaza, 2075 Kennedy Rood, Scarborough, Ontorio MIT 3V4
DISPONIBLE POUR UN EXRMEN GRATUIT DE 30 JOURS SAMS OBLICATION

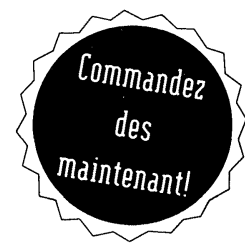

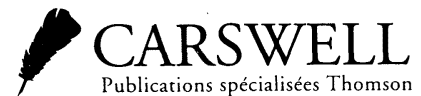

